\title{
Skeletal cavernous hemangiomas of the frontal bone with orbital roof and rim involvement
}

\author{
Bommie Florence Seo, \\ Kyo Joon Kang, \\ Sung-No Jung, \\ Jun Hee Byeon \\ Department of Plastic and \\ Reconstructive Surgery, Uijeongbu St. \\ Mary's Hospital, College of Medicine, \\ The Catholic University of Korea, \\ Uijeongbu, Korea
}

Skeletal cavernous hemangiomas are rare, benign tumors that may involve the supraorbital rim and orbital roof. However, such involvement is extremely rare. We report a case of skeletal cavernous hemangioma of the frontal bone involving the orbital roof and rim. En bloc excision and reconstruction, using a calvarial bone graft for the orbital roof and rim defect, was performed. It is important not only to perform total excision of skeletal cavernous hemangiomas, but to properly reconstruct the defects after the total excision since several complications can arise from an orbital roof and rim defect.

Keywords: Skeletal cavernous hemangioma / Orbital roof / Orbital rim / Calvarial bone graft

\section{INTRODUCTION}

Skeletal cavernous hemangiomas are benign tumors arising from the intrinsic vasculature of the bone [1], and most occur in vertebral bodies. These benign tumors account for only $0.7 \%$ of all primary bone tumors [2,3]. In addition, skeletal cavernous hemangiomas very rarely involve the supraorbital rim and orbital roof. When the tumor involves the orbital roof and rim, not only complete excision of the hemangiomas important but reconstruction of orbital roof and supraorbital rim defects created during the surgical excision is also necessary to prevent complications and maintain cosmesis.

We present a successful reconstruction using a calvarium graft after total surgical excision of skeletal cavernous hemangiomas involving the supraorbital rim and orbital roof. The patient agreed to share her experience by providing signed informed consent. Because of the retrospective nature of the study, ethics approval was not required.

\footnotetext{
Correspondence: Jun Hee Byeon

Department of Plastic and Reconstructive Surgery, Uijeongbu St. Mary's Hospital, College of Medicine, The Catholic University of Korea, 271 Cheonbo-ro, Uijeongbu 11765, Korea

E-mail: byeon@catholic.ac.kr

Received May 31, 2018 / Revised August 14, 2018 / Accepted August 27, 2018
}

\section{CASE REPORT}

A 49-year-old woman presented with a fixed, hard mass in the left supraorbital aspect of her forehead, accompanied by intermittent headaches in the frontoparietal area (Fig. 1). The patient had no history of trauma. Computed tomography revealed a protruding mass (diameter, $2.2 \mathrm{~cm}$ ) with multiple pores, in the left frontal skull and involving the orbital rim and roof (Fig. 2). En bloc excision was performed via the bicoronal approach, in collaboration with neurosurgeons. The exposed frontal sinus was obliterated using a pericranial flap. An anteriorly based pericranial flap was outlined on a bicoronal flap and the pericranial flap was elevated in the submusculoaponeurotic plane. The frontal sinus was packed with the pericranial flap. A calvarial bone graft was designed from the frontal skull and tailored to fit the defect; fixation was performed using titanium plates and screws. Hydroxyapatite cement (Mimix; Zimmer Biomet, Warsaw, IN, USA) was prepared by mixing $10 \mathrm{~g}$ of the cement powder with $6.9 \mathrm{~mL}$ of dilute citric acid. This material was then used to fill gaps and smoothen the surface (Figs. 3, 4).

The pathology results of the excised tissue confirmed the diagnosis of skeletal cavernous hemangiomas (Fig. 5). The patient recovered with no ocular complications, but reported hypesthesia of the forehead and scalp. At 12 months after the operation, 


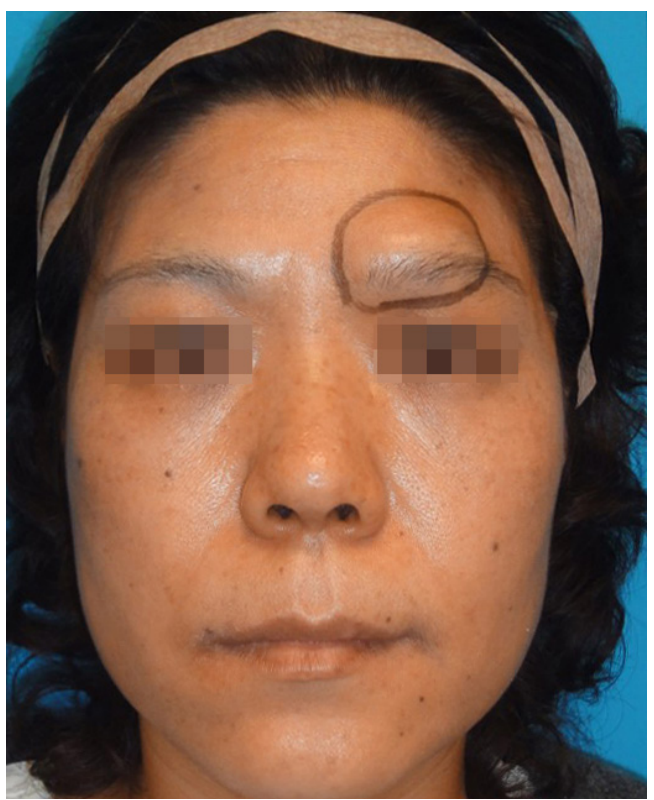

Fig. 1. A fixed, hard mass in the left supraorbital aspect of the forehead.
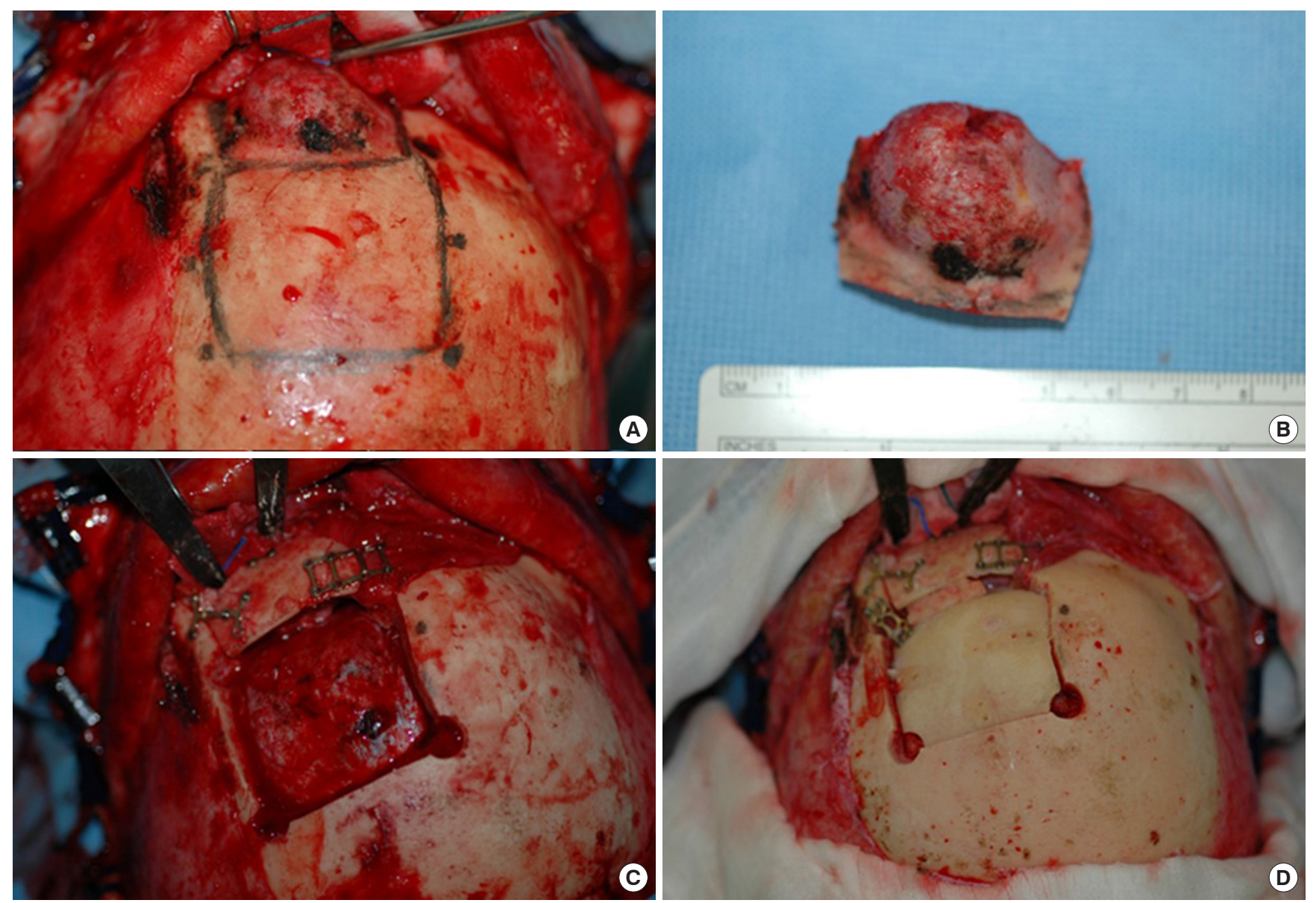

Fig. 3. The intraoperative procedure. (A) View from the vertex after bicoronal dissection and hemangioma exposure. (B) En bloc excision, including the surrounding unaffected bone. (C) Harvest of calvarial bone reconstructed to include both the orbital roof segment and supraorbital rim. (D) Reconstruction of the calvarial defect with the outer cortex of the harvested graft. 


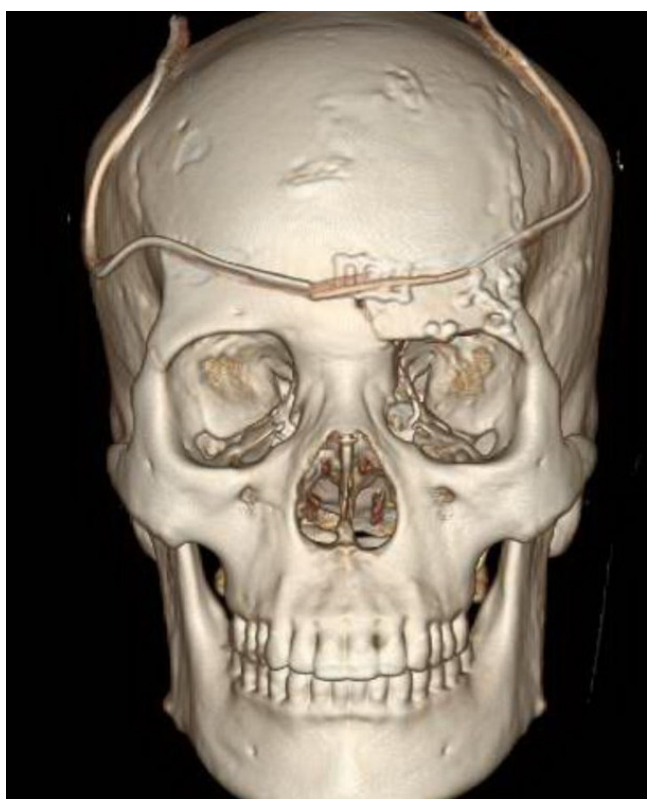

Fig. 4. Immediate postoperative three-dimensional computed tomography showing good support in the rim and occipital roof areas.

the patient reported resolution of the frontoparietal headaches and slow recovery of forehead and scalp sensations.

\section{DISCUSSION}

Skeletal cavernous hemangiomas are rare, benign tumors of blood vessels. They occur most commonly in the spinal vertebral body, followed by the skull. These lesions account for $10 \%$ of benign calvarial tumors and $0.7 \%$ of osseous neoplasms [46]. Among the bones of the skull, the lesions occur most commonly in the parietal bone, followed by the frontal bone [3,7-9].

Skeletal cavernous hemangiomas grow slowly and mainly occur in women between 20 and 40 years of age [9]. Symptoms include pain and a palpable bony hard mass that grows slowly [1]. Neurologic deficits are rare because these hemangiomas mainly grow outward rather than intracranially [6]. However, depending on the location of the lesion, neurologic deficits may appear. For example, when the tumor occurs in the temporal region, facial nerve paralysis and hearing loss may occur; vision loss and proptosis may occur when the tumor occurs in a bone involving the orbit [10].

Skeletal cavernous hemangiomas may be distinguished from eosinophilic granulomas, fibrous dysplasia, dermoid cysts, multiple myelomas, and osteoid osteomas. The pathogenesis of these lesions is not well known, but there are reports of them occurring after trauma [11]. Histologically, hemangiomas are divided into three types: cavernous, capillary, and mixed. Hem-

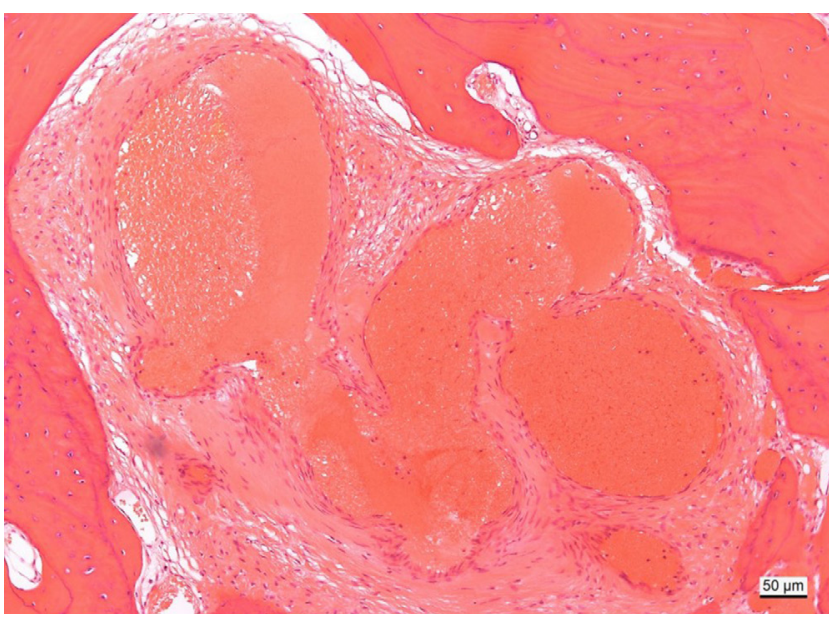

Fig. 5. Microscopic view of the specimen, cavernous hemangioma; endothelium-lined, thin-walled vascular channels interspersed among bony trabecula $(\mathrm{H} \& \mathrm{E}, \times 100)$.

angiomas occurring in the skull are mostly of the cavernous type. The cavernous type is made up of large, thin-walled vessels and sinuses lined with a single layer of endothelial cells. The capillary type is less common and is formed by small tortuous vessels. The third type involves a mixture of cavernous and capillary components $[6,12]$. Computed tomography is most useful for the diagnosis of skeletal cavernous hemangiomas, which appear as alveolate, soap bubble-like, sunburst osteolytic images and bone erosion images. Three-dimensional reconstructed computed tomography images are also useful for examining the relationship between the mass and surrounding tissues. Moreover, the normal bony tissue is replaced with the osteolytic lesion in radiology images [6].

Skeletal cavernous hemangiomas follow predominantly asymptomatic, benign courses. Therefore, it may be necessary to only observe their progress. However, surgery should be considered when there are mass effects, hemorrhages, or cosmetic abnormalities. Total surgical resection is the most preferred method of treatment. En bloc excision should include the unaffected bone margins, and reconstruction should be performed for any post-excisional defects [13-15]. If orbital roof and rim defects are not properly reconstructed, ocular symptoms, such as exophthalmos, enophthalmos, proptosis, diplopia, restricted vision, altered vision, pain, and discomfort, may occur. Traumatic encephalocele, cerebrospinal fluid leaks, and meningitis can also occur [16]. It is important to reconstruct the orbital rim according to the original contours. Methods for reconstructing the orbital roof and rim using bone grafts, high-density porous polyethylene (Medpor; Stryker, Kalamazoo, MI, USA), and titanium mesh (TiMesh; Medtronic, Minneapolis, MN, USA) exist. The ideal method for reconstructing the or- 
bital roof and rim is to bend it freely, according to the anatomic shape, to produce radiopaque properties that facilitate postoperative radiological examinations and enhance its long-term stability. A calvarium graft has the disadvantage of donor site morbidity. However, employing it is the most biocompatible and radiopaque reconstruction method. In addition, bone grafts have a smooth surface from which the periorbita can be easily dissected in a secondary reconstruction, without additional cost [17-19].

In the present patient, a calvarial bone graft was designed from the frontal skull and tailored to fit the defect. Graft fixation was performed using titanium plates and screws. Bone cement was applied to fill the gaps and smoothen the surface. The patient recovered well without ocular symptoms or distinct complications 12 months after surgery and the reconstruction produced excellent cosmesis.

In conclusion, skeletal cavernous hemangiomas are very rare, benign tumors, especially those involving the orbital roof and rim. We presented a case involving calvarial bone graft-based reconstruction after total surgical excision of a skeletal cavernous hemangioma that included the orbital rim and roof. After surgical excision, proper defect reconstruction is important.

\section{CONFLICT OF INTEREST}

No potential conflict of interest relevant to this article was reported.

\section{PATIENT CONSENT}

The patients provided written informed consent for the publication and the use of their images.

\section{REFERENCES}

1. Khanam H, Lipper MH, Wolff CL, Lopes MB. Calvarial hemangiomas: report of two cases and review of the literature. Surg Neurol 2001;55:63-7.

2. Ajja A, Oukacha N, Gazzaz M, Akhaddar A, Elmostarchid B, Kadiri B, et al. Cavernous hemangioma of the parietal bone: a case report. J Neurosurg Sci 2005;49:159-62.

3. Yoshida D, Sugisaki Y, Shimura T, Teramoto A. Cavernous hemangioma of the skull in a neonate. Childs Nerv Syst 1999; 15:351-3.
4. Murrone D, De Paulis D, Millimaggi DF, Del Maestro M, Galzio RJ. Cavernous hemangioma of the frontal bone: a case report. J Med Case Rep 2014;8:121.

5. Honda M, Toda K, Baba H, Yonekura M. Congenital cavernous angioma of the temporal bone: case report. Surg Neurol 2003;59:120-3.

6. Liu JK, Burger PC, Harnsberger HR, Couldwell WT. Primary intraosseous skull base cavernous hemangioma: case report. Skull Base 2003;13:219-28.

7. Heckl S, Aschoff A, Kunze S. Cavernomas of the skull: review of the literature 1975-2000. Neurosurg Rev 2002;25:56-62.

8. Corr P. Multiple calvarial haemangiomas. Australas Radiol 2000;44:118-20.

9. Suzuki Y, Ikeda H, Matsumoto K. Neuroradiological features of intraosseous cavernous hemangioma: case report. Neurol Med Chir (Tokyo) 2001;41:279-82.

10. Hook SR, Font RL, McCrary JA, Harper RL. Intraosseous capillary hemangioma of the frontal bone. Am J Ophthalmol 1987;103:824-7.

11. Cheng NC, Lai DM, Hsie MH, Liao SL, Chen YB. Intraosseous hemangiomas of the facial bone. Plast Reconstr Surg 2006;117: 2366-72.

12. Rios Dias GD, Velasco Cruz AA. Intraosseous hemangioma of the lateral orbital wall. Ophthalmic Plast Reconstr Surg 2004; 20:27-30

13. Sary A, Yavuzer R, Latfoglu O, Celebi MC. Intraosseous zygomatic hemangioma. Ann Plast Surg 2001;46:659-60.

14. Koybasi S, Saydam L, Kutluay L. Intraosseous hemangioma of the zygoma. Am J Otolaryngol 2003;24:194-7.

15. Konior RJ, Kelley TF, Hemmer D. Intraosseus zygomatic hemangioma. Otolaryngol Head Neck Surg 1999;121:122-5.

16. Martello JY, Vasconez HC. Supraorbital roof fractures: a formidable entity with which to contend. Ann Plast Surg 1997;38: 223-7.

17. Haug RH, Van Sickels JE, Jenkins WS. Demographics and treatment options for orbital roof fractures. Oral Surg Oral Med Oral Pathol Oral Radiol Endod 2002;93:238-46.

18. Manolidis S, Weeks BH, Kirby M, Scarlett M, Hollier L. Classification and surgical management of orbital fractures: experience with 111 orbital reconstructions. J Craniofac Surg 2002; 13:726-37.

19. Mohindra S, Mukherjee KK, Chhabra R, Gupta R. Orbital roof growing fractures: a report of four cases and literature review. Br J Neurosurg 2006;20:420-3. 